Conclusions: SSc-patients have markedly reduced muscle endurance and muscle function compared with reference values and patients with moderate-endstage lung disease have reduced lower extremity muscle function compared to patients with no-mild lung disease. SSc-patients have slightly less AROM than controls and dcSSc-patients are more restricted than IcSSc. This study highlights the importance of identifying patients with an enhanced risk of developing severe muscle impairments. We suggest that physiotherapists regularly assess muscle function using standardised tests and when needed initiate exercise to improve function

Disclosure of Interest: None declared

DOI: 10.1136/annrheumdis-2018-eular.2575

\section{THU0718-HPR THE PSYCHOSOCIAL IMPACT OF JOINT HYPERMOBILITY SYNDROMEAND EHLERS-DANLOS SYNDROME (HYPERMOBILITY TYPE): A QUALITATIVE INTERVIEW STUDY}

S.E. Bennett, S. Palmer, N. Walsh, T. Moss. Faculty of Health and Applied Sciences, The University of the West of England, Bristol, UK

Background: Existing research examining those with Joint Hypermobility Syndrome (JHS) and Ehlers-Danlos Syndrome (Hypermobility Type) (EDS-HT) has predominately focused on factors such as pain, range of movement and physical function. However psychosocial factors have received much less attention.

Objectives: This study sought to 1. Identify the psychosocial impact of JHS/EDSHT by examining participants' lived experiences, and; 2 . Identify characteristics of effective coping with JHS/EDS-HT, using qualitative methods.

Methods: Adults with JHS/EDS-HT took part in semi-structured telephone interviews to discuss their own lived experiences and the impact of the condition on their lives. All met the Hakim and Grahame (2003) five-item criteria for clinically significant joint hypermobility, and had a self-confirmed diagnosis of JHS/EDSHT. The transcripts were coded using NVivo 10 and analysed using inductive thematic analysis.

Results: 17 participants (14 women, 3 men) took part (age range 22-70, mean 38 years). The sample was purposively selected from across the UK to broadly represent different genders, ages and ethnicities. Inductive thematic analysis indicated five main themes:

Healthcare limitations: All participants reported a lack of awareness of JHS/EDS HT among healthcare professionals, and diagnosis typically took several years. Examples were given where local anaesthetics had either partly or completely failed, leaving patients aware of severe pain during surgical or dental procedures. $A$ restricted life: Participants experienced a range of symptoms including joint pain and instability, fatigue, gastrointestinal issues, frequent dislocations and subluxations. Due to difficulty completing daily activities, some relied on their partners or family for support, but this led to feelings of guilt and shame.

Social stigma: The invisible nature of their condition led to participants facing criticism and confrontations with others as they 'looked fine'. Fears of being judged led some to hide their symptoms. Many felt frustrated and angry that due to fatigue or injury they could not keep up with friends, family or colleagues.

Fear of the unknown: Not knowing when the next injury was going to occur, and how JHS/EDS-HT would affect them over time made participants especially fearful of declines in their physical ability. Many cited of a lack of reliable information about their condition, other than in published books or research journals. Psychological support to better cope with the enduring impact of JHS/EDS-HT on their lives was lacking.

Ways of coping: Several coping approaches were identified by participants, including acceptance of their condition, building social networks, finding out more about JHS/EDS-HT and adapting their activities. Physiotherapists were instrumental in supporting participants to exercise regularly.

Conclusions: The results of this qualitative study highlight the significant psychosocial impact of JHS/EDS-HT on participants' lives. Further research should consider potential interventions to improve information provision, address psychological support and increase awareness of JHS/EDS-HT among healthcare professionals.

\section{REFERENCE:}

[1] Hakim A, Grahame R. A simple questionnaire to detect hypermobility: An adjunct to the assessment of patients with diffuse musculoskeletal pain. International Journal of Clinical Practice 2003;57(3):163-166.

Disclosure of Interest: None declared

DOI: 10.1136/annrheumdis-2018-eular.2107

\section{THU0719-HPR NON-PHARMACOLOGICAL CARE IN SYSTEMIC SCLEROSIS: ROOM FOR IMPROVEMENT?}

J.K. Stöcker ${ }^{1}$, J. Spierings ${ }^{2}$, C.H.M. van den Ende ${ }^{1}$, M.C. Vonk ${ }^{1}$, on behalf of ARCH Study Group. ${ }^{1}$ Rheumatology, Radboudumc, Nijmegen; ${ }^{2}$ Rheumatology, UMC Utrecht, Utrecht, Netherlands

Background: Although health professional (HP) treatment is considered to be a corner stone in the management of systemic sclerosis (SSc), little is known about the referral process to and the content of non-pharmacological care in SSc.

Objectives: To describe the contribution of HP to SSc care from the perspective of SSc patients, including the referral process, use of care provided by HP, treatment targets and outcome satisfaction

Methods: Dutch SSc patients from different hospitals were invited through their rheumatologists (RT) to complete an still ongoing online survey provided by the $\mathrm{ARCH}$ (Arthritis Research and Collaboration Hub) working group, containing questions on a wide range of issues within SSc care. Items concerning access to care (referral versus direct access), use of HP services, and quality of care perceived by patients assessed by the $\mathrm{CQ}$ Index were extracted to answer the research question. Reasons for referral/direct access and treatment targets were examined by means of open questions.

Results: On January 10th, 2018, 433 SSc patients, $73 \%$ women $(n=317)$, with a median age of $61(S D=10,81)$ completed the survey. $38 \%$ of the patients had limited and $23 \%$ diffuse cutaneous SSc. The mean time since diagnosis was 4,1 years.

In total, 10 different HP disciplines were reported and $76 \%(n=331)$ of the patients ever had contact with one or more HP; $50 \%(n=215)$ had contact with a HP in the past year. Compared to the other 9 disciplines named, physiotherapists (PT) were the most often referred to; $57 \%(n=245)$ had PT treatment since SSc onset, $41 \%$ $(n=177)$ in 2017, whereas only 35 were referred to occupational therapists and 9 to hand therapists (figure 1)

Rheumatologists (RT) were the main referrers to HP care with $49 \%(n=106) ; 27 \%$ $(n=59)$ sought care by one or more HP through direct access in the past year. A total of $52 \%(n=111)$ of the 215 patients that received HP treatment in 2017 perceived the collaboration between their RT and the treating HP as insufficient and $68 \%(n=146)$ reported that RT and HP did not make good agreements with each other. Further, $45 \%(n=97)$ of the patients felt that there was no coordination between the referrers and the HP about advices issued.

$76 \%(n=164)$ of respondents found their HP sufficiently competent for SSc treatment. $73 \%(n=156)$ could cope better with their complaints after the treatment and reported improvement in their daily activities. Qualitative analysis of the data on the PT as main HP treatment provider in 2017 yielded 36 different referral reasons and 42 treatment targets, of which the most frequently mentioned $(>4)$ are shown below. Most frequent reasons for referral and therapeutic objectives physio therapy Referral reasons: pain, joint problems, shoulder problems, decreased mobility, back problems, muscle weakness/stiffness/ pain and general stiffness.

Treatment targets: improvement of general mobility, joint mobility, condition, muscle function, skin flexibility and hand function, pain relief, stiffness avoidance and fatigue reduction.

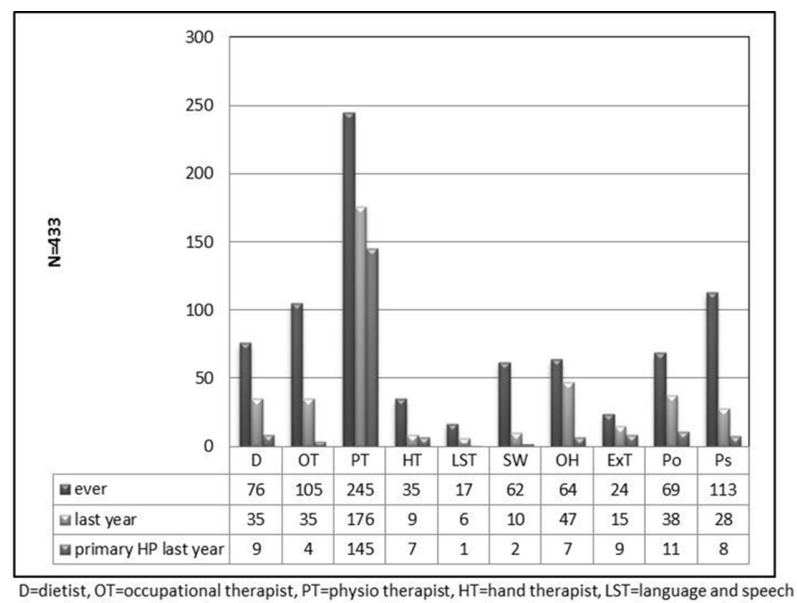

Derast $\mathrm{SW}=$ =

Abstract THU0719HPR - Figure 1. Frequency of referrals to health professionals

Conclusions: HP treatment is a meaningful part of SSc care, as reflected in the satisfaction of SSc patients. The results describe a suboptimal communication 